\title{
Volume preserving diffeomorphisms with orbital shadowing
}

\author{
Keonhee Lee ${ }^{1}$ and Manseob Lee ${ }^{2^{*}}$
}

${ }^{\text {"Correspondence: }}$

Imsds@mokwon.ac.kr

²Department of Mathematics, Mokwon University, Daejeon,

302-729, Korea

Full list of author information is

available at the end of the article

\begin{abstract}
Let $f$ be a volume-preserving diffeomorphism of a closed $C^{\infty}$ Riemannian manifold $M$. In this paper, we show that the following are equivalent:

(a) $f$ belongs to the $C^{1}$-interior of the set of volume-preserving diffeomorphisms with orbital shadowing,

(b) $f$ is Anosov.
\end{abstract}

MSC: Primary 37C50; secondary 34D10

Keywords: normally hyperbolic; hyperbolic; orbital shadowing; shadowing; Anosov

\section{Introduction}

A fundamental problem in differentiable dynamical systems is to understand how a robust dynamic property on the underlying manifold would influence the behavior of the tangent map on the tangent bundle. For instance, in [1], Mañé proved that any $C^{1}$ structurally stable diffeomorphism is an Axiom $A$ diffeomorphism. And in [2], Palis extended this result to $\Omega$-stable diffeomorphisms. Let $M$ be a closed $C^{\infty}$ Riemannian manifold endowed with a volume form $\omega$. Let $\mu$ denote the Lebesgue measure associated to $\omega$, and let $d$ denote the metric induced on $M$ by the Riemannian structure. Denote by $\operatorname{Diff}_{\mu}(M)$ the set of diffeomorphisms which preserves the Lebesgue measure $\mu$ endowed with the $C^{1}$-topology. We know that every volume preserving diffeomorphism satisfying Axiom $A$ is Anosov (for more details, see [3]).

For $\delta>0$, a sequence of points $\left\{x_{i}\right\}_{i=a}^{b}(-\infty \leq a<b \leq \infty)$ in $M$ is called a $\delta$-pseudo-orbit of $f$ if $d\left(f\left(x_{i}\right), x_{i+1}\right)<\delta$ for all $a \leq i \leq b-1$. Let $\Lambda \subset M$ be a closed $f$-invariant set. We say that $f$ has the shadowing property on $\Lambda$ (or $\Lambda$ is shadowable) if for every $\epsilon>0$, there is $\delta>0$ such that for any $\delta$-pseudo-orbit $\left\{x_{i}\right\}_{i=a}^{b} \subset \Lambda$ of $f(-\infty \leq a<b \leq \infty)$, there is a point $y \in M$ such that $d\left(f^{i}(y), x_{i}\right)<\epsilon$ for all $a \leq i \leq b-1$. It is easy to see that $f$ has the shadowing property on $\Lambda$ if and only if $f^{n}$ has the shadowing property on $\Lambda$ for $n \in \mathbb{Z} \backslash\{0\}$. The notion of pseudo-orbits often appears in several methods of the modern theory of dynamical systems. Moreover, the shadowing property plays an important role in the investigation of stability theory. In fact, Pilyugin [4] and Robinson [5] showed that if a diffeomorphism $f$ is structurally stable, then $f$ has the shadowing property. Moreover, Sakai [6] proved that if there is a $C^{1}$-neighborhood $\mathcal{U}(f)$ of $f$ such that for any $g \in \mathcal{U}(f), g$ has the shadowing property, then $f$ is structurally stable. For each $x \in M$, let $\mathcal{O}_{f}(x)$ be the orbit of $f$ through $x$; that is,

$$
\mathcal{O}_{f}(x)=\left\{f^{n}(x): n \in \mathbb{Z}\right\}
$$

C 2013 Lee and Lee; licensee Springer. This is an Open Access article distributed under the terms of the Creative Commons Attribution License (http://creativecommons.org/licenses/by/2.0), which permits unrestricted use, distribution, and reproduction in any medium, provided the original work is properly cited. 
We say that $f$ has the orbital shadowing property on $\Lambda$ (or $\Lambda$ is orbitally shadowable) if for any $\epsilon>0$, there exists $\delta>0$ such that for any $\delta$-pseudo-orbit $\xi=\left\{x_{i}\right\}_{i \in \mathbb{Z}} \subset \Lambda$, we can find a point $y \in M$ such that

$$
\mathcal{O}_{f}(y) \subset B_{\epsilon}(\xi) \text { and } \xi \subset B_{\epsilon}\left(\mathcal{O}_{f}(y)\right)
$$

where $B_{\epsilon}(A)$ denotes the $\epsilon$-neighborhood of a set $A \subset M . f$ is said to have the weak shadowing property on $\Lambda$ (or $\Lambda$ is weakly shadowable) if for any $\epsilon>0$, there exists $\delta>0$ such that for any $\delta$-pseudo-orbit $\xi=\left\{x_{i}\right\}_{i \in \mathbb{Z}} \subset \Lambda$, there is a point $y \in M$ such that $\xi \subset B_{\epsilon}\left(\mathcal{O}_{f}(y)\right)$. Note that if $f$ has the shadowing property, then $f$ has the orbital shadowing property, but the converse is not true (see [7]). It is easy to see that $f$ has the orbital shadowing property on $\Lambda$ if and only if $f^{n}$ has the orbital shadowing property on $\Lambda$ for $n \in \mathbb{Z} \backslash\{0\}$.

We say that $\Lambda$ is hyperbolic if the tangent bundle $T_{\Lambda} M$ has a $D f$-invariant splitting $E^{s} \oplus$ $E^{u}$ and there exist constants $C>0$ and $0<\lambda<1$ such that

$$
\left\|\left.D_{x} f^{n}\right|_{E_{x}^{s}}\right\| \leq C \lambda^{n} \quad \text { and } \quad\left\|\left.D_{x} f^{-n}\right|_{E_{x}^{u}}\right\| \leq C \lambda^{-n}
$$

for all $x \in \Lambda$ and $n \geq 0$.

We denote by $\mathcal{F}_{\mu}(M)$ the set of diffeomorphisms $f \in \operatorname{Diff}_{\mu}(M)$ which have a $C^{1}$ neighborhood $\mathcal{U}(f) \subset \operatorname{Diff}_{\mu}(M)$ such that for any $g \in \mathcal{U}(f)$, every periodic point of $g$ is hyperbolic.

Very recently, Arbieto and Catalan [3] proved that every volume preserving diffeomorphism in $\mathcal{F}_{\mu}(M)$ is Anosov. To prove this, they used Mañés results in [1, Proposition II.1] and showed that $\overline{P(f)}$ is hyperbolic if $f \in \mathcal{F}_{\mu}(M)$. Thus, we have the following theorem.

Theorem 1.1 [3, Theorem 1.1] Every diffeomorphism in $\mathcal{F}_{\mu}(M)$ is Anosov.

Let int $\mathcal{O S}_{\mu}(M)$ denote the $C^{1}$-interior of the set of volume preserving diffeomorphisms in $\operatorname{Diff}_{\mu}(M)$ satisfying the orbital shadowing property. In [7], the authors proved that the $C^{1}$-interior of the set of diffeomorphisms having the orbital shadowing property coincides with the set of structurally stable diffeomorphisms. Note that if a diffeomorphism satisfies structurally stable then it is not Anosov in general. But the converse is true. Finally, we prove the following theorem.

Theorem 1.2 The set $\mathcal{A N}_{\mu}(M)$ of Anosov diffeomorphisms in $\operatorname{Diff}_{\mu}(M)$ coincides with the $C^{1}$-interior of the set of diffeomorphisms in $\operatorname{Diff}_{\mu}(M)$ with orbital shadowing; that is, $\mathcal{A N}_{\mu}(M)=\operatorname{int} \mathcal{O S}_{\mu}(M)$.

\section{Proof of Theorem 1.2}

Remark 2.1 Let $f \in \operatorname{Diff}_{\mu}^{1}(M)$. From Moser's theorem (see [8]), we can find a smooth conservative change of coordinates $\varphi_{x}: U(x) \rightarrow T_{x} M$ such that $\varphi_{x}(x)=0$, where $U(x)$ is a small neighborhood of $x \in M$.

Recall that $f$ has the orbital shadowing property on $\Lambda$ ( $\Lambda$ is orbitally shadowable) if for any $\epsilon>0$, there is $\delta>0$ such that for any $\delta$-pseudo orbit $\xi=\left\{x_{i}\right\}_{i \in \mathbb{Z}} \subset \Lambda$ of $f$, there is $\in M$ such that

$$
\mathcal{O}_{f}(y) \subset B_{\epsilon}(\xi) \text { and } \xi \subset B_{\epsilon}\left(\mathcal{O}_{f}(y)\right)
$$


Notice that in this definition, only $\delta$-pseudo orbits of $f$ are contained in $\Lambda$, but the shadowing point $y \in M$ is not necessarily contained in $\Lambda$. To prove our result, we use Franks' lemma which is proved in [9, Proposition 7.4].

Lemma 2.2 Let $f \in \operatorname{Diff}_{\mu}^{1}(M)$, and $\mathcal{U}$ be a $C^{1}$-neighborhood off in $\operatorname{Diff}_{\mu}^{1}(M)$. Then there exist a $C^{1}$-neighborhood $\mathcal{U}_{0} \subset \mathcal{U}$ of $f$ and $\epsilon>0$ such that if $g \in \mathcal{U}_{0}$, any finite $f$-invariant set $E=\left\{x_{1}, \ldots, x_{m}\right\}$, any neighborhood $U$ of $E$, and any volume-preserving linear maps $L_{j}: T_{x_{j}} M \rightarrow T_{g\left(x_{j}\right)} M$ with $\left\|L_{j}-D_{x_{j}} g\right\| \leq \epsilon$ for all $j=1, \ldots, m$, there is a conservative diffeomorphism $g_{1} \in \mathcal{U}$ coinciding with $f$ on $E$ and out of $U$, and $D_{x_{j}} g_{1}=L_{j}$ for all $j=1, \ldots, m$.

We introduce the notion of normally hyperbolic which was founded in [10]. Let $V \subset M$ be an invariant submanifold of $f \in \operatorname{Diff}_{\mu}(M)$. We say that $V$ is normally hyperbolic if there is a splitting $T_{V} M=T V \oplus N^{s} \oplus N^{u}$ such that

- the splitting depends continuously on $x \in V$,

- $D_{x} f\left(N_{x}^{\sigma}\right)=N_{f(x)}^{\sigma}(\sigma=s, u)$ for all $x \in V$,

- there are constants $C>0$ and $0<\lambda<1$ such that for every unit vector $x \in T_{x} V$, $v^{s} \in N_{x}^{s}$ and $v^{u} \in N_{x}^{u}(x \in V)$, we have

$$
\left\|D_{x} f^{n}\left(v^{s}\right)\right\| \leq C \lambda^{n}\left\|D_{x} f^{n}(v)\right\| \quad \text { and } \quad\left\|D_{x} f^{n}\left(v^{u}\right)\right\| \geq C^{-1} \lambda^{-1}\left\|D_{x} f^{n}(v)\right\|
$$

for all $n \geq 0$.

Proposition 2.3 If $\in \operatorname{int} \mathcal{O S}(M)$, then every periodic point off is hyperbolic.

Proof Take $f \in \operatorname{int} \mathcal{O S}_{\mu}(M)$, and $\mathcal{U}(f)$ is a $C^{1}$-neighborhood of $f \in \operatorname{Diff}_{\mu}(M)$. Let $\epsilon>0$ and $\mathcal{V}(f) \subset \mathcal{U}_{0}(f)$ be the number and $C^{1}$-neighborhood of $f$ corresponding to $\mathcal{U}(f)$ given by Lemma 2.2. To derive a contradiction, we assume that there exists a nonhyperbolic periodic point $p \in P(g)$ for some $g \in \mathcal{V}(f)$. To simplify the notation in the proof, we may assume that $g(p)=p$. Then there is at least one eigenvalue $\lambda$ of $D_{p} g$ such that $|\lambda|=1$.

By making use of Lemma 2.2, we linearize $g$ at $p$ using Moser's theorem; that is, by choosing $\alpha>0$ sufficiently small, we construct $g_{1} C^{1}$-nearby $g$ such that

$$
g_{1}(x)= \begin{cases}\varphi_{p}^{-1} \circ D_{p} g \circ \varphi_{p}(x) & \text { if } x \in B_{\alpha}(p), \\ g(x) & \text { if } x \notin B_{4 \alpha}(p) .\end{cases}
$$

Then $g_{1}(p)=g(p)=p$.

First, we may assume that $\lambda \in \mathbb{R}$ with $\lambda=1$. Let $v$ be the associated non-zero eigenvector such that $\|v\|=\alpha / 4$. Then we can get a small arc

$$
\mathcal{I}_{v}=\{t v:-1 \leq t \leq 1\} \subset \varphi_{p}\left(B_{\alpha}(p)\right) .
$$

Take $\epsilon=\alpha / 8$. Let $0<\delta<\epsilon$ be a number of the orbital shadowing property of $g_{1}$ corresponding to $\epsilon$. Then by our construction of $g_{1}$,

$$
\varphi_{p}^{-1}\left(\mathcal{I}_{v}\right) \subset B_{\alpha}(p)
$$


Then it is clear that $\varphi_{p}^{-1}\left(\mathcal{I}_{v}\right)$ is normally hyperbolic for $g_{1}$. Put $\mathcal{J}_{p}=\varphi_{p}^{-1}\left(\mathcal{I}_{v}\right)$. Given a constant $\delta>0$, we construct a $\delta$-pseudo orbit $\xi=\left\{x_{i}\right\}_{i \in \mathbb{Z}} \subset \mathcal{J}_{p}$ as follows. For fixed $k \in \mathbb{Z}$, choose distinct points $x_{0}=p, x_{1}, x_{2}, \ldots, x_{k}$ in $\mathcal{J}_{p}$ such that

(a) $d\left(x_{i}, x_{i+1}\right)<\delta$ for $i=0,1, \ldots, k-1$,

(b) $d\left(x_{-i-1}, x_{-i}\right)<\delta$ for $i=0, \ldots, k-1$,

(c) $x_{0}=x$ and $d\left(x_{-k}, x_{k}\right)>2 \epsilon$.

Now, we define $\xi=\left\{x_{i}\right\}_{i \in \mathbb{Z}}$ by $x_{k i+j}=x_{j}$ for $i \in \mathbb{Z}$ and $j=-k-1,-k-2, \ldots,-1,0,1, \ldots, k-1$. Since $g_{1}$ has the orbital shadowing property, $\left.g_{1}\right|_{\mathcal{J}_{p}}$ must have the orbital shadowing property. Thus, we can find a point $y \in M$ such that $\xi \subset B_{\epsilon}\left(\mathcal{O}_{g_{1}}(y)\right)$, and $\mathcal{O}_{g_{1}}(y) \subset B_{\epsilon}(\xi)$. For any $v \in \mathcal{I}_{v}, \varphi_{p}^{-1}(v) \in \mathcal{J}_{p} \subset B_{\alpha}(p)$ and

$$
g_{1}\left(\varphi_{p}^{-1}(v)\right)=\varphi_{p}^{-1} \circ D_{p} g \circ \varphi_{p}\left(\varphi_{p}^{-1}(v)\right)
$$

Then $g_{1}\left(\varphi_{p}^{-1}(v)\right)=\varphi_{p}^{-1}(v)$. Thus, $g_{1}^{l}\left(\mathcal{J}_{p}\right)=\mathcal{J}_{p}$ for some $l>0$. Now, we show that if $\mathcal{J}_{p}$ is normally hyperbolic for $g_{1}$, then the shadowing points belong to $\mathcal{J}_{p}$. Assume that there is a shadowing point $y \in M \backslash \mathcal{J}_{p}$. Then by the hyperbolicity, there are $l, k \in \mathbb{Z}$ such that $d\left(g_{1}^{l}(y), x_{k}\right)>\epsilon$, where $x_{k} \in \xi=\left\{x_{i}\right\}_{i \in \mathbb{Z}}$. This is a contradiction since $\left.g_{1}\right|_{\mathcal{J}_{p}}$ has the orbital shadowing property. Thus, if $\mathcal{J}_{p}$ is normally hyperbolic for $g_{1}$, then the shadowing point belongs to $\mathcal{J}_{p}$. Since $\left.g_{1}\right|_{\mathcal{J}_{p}}$ has the orbital shadowing property, from the above facts, we have $y \in \mathcal{J}_{p}$. But $g_{1}^{l}\left(\mathcal{J}_{p}\right)=\mathcal{J}_{p}$ and so $\left.g_{1}^{l}\right|_{\mathcal{J}_{p}}$ is the identity map. Then $\left.g_{1}^{l}\right|_{\mathcal{J}_{p}}$ does not have the orbital shadowing property. Thus, $\left.g_{1}\right|_{\mathcal{J}_{p}}$ also does not have the orbital shadowing property.

Finally, if $\lambda \in \mathbb{C}$, then to avoid the notational complexity, we may assume that $g(p)=p$. As in the first case, by Lemma 2.2, there are $\alpha>0$ and $g_{1} \in \mathcal{V}(f)$ such that $g_{1}(p)=g(p)=p$ and

$$
g_{1}(x)= \begin{cases}\varphi_{p}^{-1} \circ D_{p} g \circ \varphi_{p}(x) & \text { if } x \in B_{\alpha}(p), \\ g(x) & \text { if } x \notin B_{4 \alpha}(p) .\end{cases}
$$

With a $C^{1}$-small modification of the map $D_{p} g$, we may suppose that there is $l>0$ (the minimum number) such that $D_{p} g^{l}(v)=v$ for any $v \in \varphi_{p}\left(B_{\alpha}(p)\right) \subset T_{p} M$. Then we can go on with the previous argument in order to reach the same contradiction. Thus, every periodic point of $f \in \operatorname{int} \mathcal{O S}_{\mu}(M)$ is hyperbolic.

End of the proof of Theorem 1.2 Let $f \in \operatorname{int} \mathcal{O S}_{\mu}(M)$. By Proposition 2.3, we see that $f \in$ $\mathcal{F}_{\mu}(M)$. Thus, by Theorem 1.1, $f$ is Anosov. 


\section{Acknowledgements}

We wish to thank the referee for carefully reading of the manuscript and providing us many good suggestions. $\mathrm{KL}$ is supported by the National Research Foundation (NRF) of Korea funded by the Korean Government (No. 2011-0015193). ML is supported by the Basic Science Research Program through the National Research Foundation of Korea (NRF) funded by the Ministry of Education, Science and Technology, Korea (No. 2011-0007649).

Received: 12 July 2012 Accepted: 26 December 2012 Published: 14 January 2013

\section{References}

1. Mãné, R: An ergodic closing lemma. Ann. Math. 116, 503-540 (1982)

2. Palis, J: On the $C^{l} \Omega$-stability conjecture. Publ. Math. Inst. Hautes Études Sci. 66, 211-215 (1988)

3. Arbieto, A, Catalan, T: Hyperbolicity in the volume preserving scenario. Ergodic Theory Dyn. Syst. (2012). doi:10.1017/etds.2012.111

4. Pilyugin, SY: Shadowing in Dynamical Systems. Lecture Notes in Math., vol. 1706. Springer, Berlin (1999)

5. Robinson, C: Stability theorems and hyperbolicity in dynamical systems. Rocky Mt. J. Math. 7, 425-437 (1977)

6. Sakai, K: Pseudo orbit tracing property and strong transversality of diffeomorphisms on closed manifolds. Osaka J. Math. 31, 373-386 (1994)

7. Pilyugin, SY, Rodionova, AA, Sakai, K: Orbital and weak shadowing properties. Discrete Contin. Dyn. Syst. 9, 287-308 (2003)

8. Moser, J: On the volume elements on a manifold. Trans. Am. Math. Soc. 120, 286-294 (1965)

9. Bonatti, C, Díaz, LJ, Pujals, ER: A C $C^{1}$-generic dichotomy for diffeomorphisms: weak forms of hyperbolicity or infinitely many sinks or sources. Ann. Math. 158, 355-418 (2003)

10. Hirsh, M, Pugh, C, Shub, M: Invariant Manifolds. Lecture Notes in Math. Springer, Berlin (1977)

doi:10.1186/1029-242X-2013-18

Cite this article as: Lee and Lee: Volume preserving diffeomorphisms with orbital shadowing. Journal of Inequalities and Applications 2013 2013:18

\section{Submit your manuscript to a SpringerOpen ${ }^{\ominus}$ journal and benefit from:}

- Convenient online submission

- Rigorous peer review

- Immediate publication on acceptance

- Open access: articles freely available online

- High visibility within the field

- Retaining the copyright to your article 